\title{
Phase I study of 5-fluorouracil, leucovorin and bevacizumab in combination with radiation therapy in patients with locally advanced rectal cancer
}

\author{
YASUHIRO INOUE, MASATO OKIGAMI, AYA KAWAMOTO, YOSHINAGA OKUGAWA, JUNICHIRO HIRO, \\ SUSUMU SAIGUSA, YUJI TOIYAMA, KOJI TANAKA, YASUHIKO MOHRI and MASATO KUSUNOKI \\ Department of Gastrointestinal and Pediatric Surgery, Division of Reparative Medicine, Institute of Life Sciences, \\ Mie University Graduate School of Medicine, Tsu, Mie 514-8507, Japan
}

Received November 19, 2012; Accepted February 5, 2013

DOI: $10.3892 / \mathrm{mco} .2013 .78$

\begin{abstract}
A phase I clinical study was conducted to determine the maximum tolerated dose (MTD) and recommended dose (RD) of the standard treatment of 5-fluorouracil/1-leucovorin (5-FU/LV) with bevacizumab, in combination with radiation therapy in patients with locally advanced rectal cancer. Eligible patients had previously untreated stage T3 or T4 locally advanced rectal cancer. Patients received radiotherapy to the pelvis, at a total dose of 45 Gy in 25 fractions. During radiotherapy, patients received three courses of a simplified LV and 5-FU regimen (sLV5FU2), in combination with bevacizumab. Bevacizumab was infused at a fixed dose of $5 \mathrm{mg}$ / $\mathrm{kg}$ on Days 1, 15 and 29. The sLV5FU2 regimen consisted of $200 \mathrm{mg} / \mathrm{m}^{2}$ of LV administered by continuous intravenous (i.v.) infusion over $2 \mathrm{~h}$, followed by $400 \mathrm{mg} / \mathrm{m}^{2}$ of 5 -FU administered by i.v. bolus injection, delivered at an initial loading dose of $2,000 \mathrm{mg} / \mathrm{m}^{2}$ over $46 \mathrm{~h}$. The dose was gradually increased to determine the MTD and RD of this regimen. Of the patients enrolled in the study, two developed Grade 3 diarrhea and one developed Grade 3 neutropenia. Since dose-limiting toxicity (DLT) occurred in two out of the six patients, 5-FU at a dose of $2,000 \mathrm{mg} / \mathrm{m}^{2}$ over $46 \mathrm{~h}$ was determined as the MTD and designated as the RD, taking into consideration the toxicities in matched patients who received standard preoperative chemoradiotherapy with sLV5FU2 during the same period. The combination of 5-FU, LV, bevacizumab and radiotherapy in patients with locally advanced rectal cancer was found to be tolerable, with encouraging response rates. Further investigation is required in a phase II setting.
\end{abstract}

Correspondence to: Dr Yasuhiro Inoue, Department of Gastrointestinal and Pediatric Surgery, Division of Reparative Medicine, Institute of Life Sciences, Mie University Graduate School of Medicine, 2-174 Edobashi, Tsu, Mie 514-8507, Japan

E-mail: yasinoue@clin.medic.mie-u.ac.jp

Key words: preoperative chemoradiotherapy, bevacizumab, rectal cancer

\section{Introduction}

The management of rectal cancer has evolved over the past decades, with improvements in surgical techniques and adjuvant therapy. Studies involving advanced rectal cancer have demonstrated that preoperative or postoperative radiotherapy significantly reduces the risk of local recurrence and cancer-specific mortality, compared to surgery alone $(1,2)$. Furthermore, the addition of 5-fluorouracil (5-FU) to preoperative radiotherapy has been shown to improve the pathological complete response rate and locoregional control, compared to radiotherapy alone $(3,4)$. Thus, preoperative radiotherapy and concurrent 5-FU administration has become a standard treatment in locally advanced rectal cancer. However, disease recurrence remains the major cause of mortality in these patients and recent efforts, involving the incorporation of newer cytotoxic or molecular-targeted agents into chemoradiotherapy regimens, have been reported to improve oncological outcome. The addition of oxaliplatin or irinotecan to 5-FU administered concurrently with radiotherapy, resulted in a more favorable pathological response in certain phase I/II trials $(5,6)$, while the addition of other chemotherapeutic drugs significantly increased toxicity or exhibited no oncological improvement $(7,8)$. Similarly, incorporating anti-vascular endothelial growth factor (VEGF) or anti-epidermal growth factor receptor therapies into preoperative chemoradiotherapy in patients with locally advanced rectal cancer did not significantly enhance pathological downstaging, although few randomized trials have been undertaken (9).

Our previous study demonstrated that preoperative chemoradiotherapy enhanced tumor-specific VEGF expression, particularly in individual cancer cells in vitro and in vivo (10). We also demonstrated that VEGF gene expression in pre-irradiation tumor biopsies may be a useful marker in predicting tumor responsiveness to preoperative chemoradiotherapy, as well as disease recurrence (11). Subsequently, we hypothesized that dose-intensified treatment strategies, including the anti-VEGF antibody, in a preoperative setting, may be an ideal adjuvant treatment. This is attributed to the fact that angiogenesis is required for tumor growth and malignant progression and VEGF is a crucial regulator of this process. However, 
there have been no studies on chemoradiotherapy with bevacizumab as preoperative treatment in Japanese patients with locally advanced rectal cancer, although more active preoperative chemoradiotherapy, using bevacizumab, oxaliplatin and fluoropyrimidine for locally advanced rectal cancer, has already been reported in Western countries $(12,13)$. The present clinical phase I study was conducted to determine the maximum tolerated dose (MTD) and recommended dose (RD) of standard 5-FU/1-leucovorin (5-FU/LV) with bevacizumab and radiation in Japanese patients with locally advanced rectal cancer. We also evaluated adverse effects caused by the treatment and resectability of locally advanced rectal cancer, including pathological response rates.

\section{Patients and methods}

Ethics and patient consent. The present study was reviewed and approved by the Mie University Institutional Review Board and the study was performed in accordance with the Helsinki Declaration of 1975, as revised in 2000. Patients were required to provide written informed consent prior to enrollment.

Eligibility criteria. Eligible patients had previously untreated stage T3 or T4 locally advanced rectal cancer, as determined by computed tomography (CT), magnetic resonance imaging (MRI) or endoscopic ultrasound criteria and had histologically confirmed adenocarcinoma prior to surgery. Eligible patients also had an Eastern Cooperative Oncology Group (ECOG) performance status of 0-1 and a survival expectation of $>3$ months. Additional eligibility criteria included: age 20-80 years at enrollment; no severe compromise of main organ functions (including bone marrow, lung, liver and kidney) or blood biochemistry (leukocyte count 4,000-12,000/. $\mathrm{mm}^{3}$; platelet count $\geq 100 \times 10^{3} / \mu \mathrm{l}$; hemoglobin concentration $\geq 9.0 \mathrm{~g} / \mathrm{dl}$; total bilirubin concentration $\leq 1.5 \mathrm{x}$ upper limit of normal; serum aspartate aminotransferase and alanine aminotransferase levels $<2.5 \mathrm{x}$ upper limit of normal; and serum creatinine concentration $<1.5 \mathrm{x}$ upper limit of normal).

The exclusion criteria were patients with potential risk factors for bevacizumab, 5-FU and LV-related adverse events. The risk factors included: patients with child-bearing potential or lactation; clinical evidence of thrombosis, requiring medication; uncontrolled hypertension; clinically significant cardiovascular or pulmonary disease; clinical evidence of gastrointestinal bleeding; bowel obstruction and perforation; active inflammatory bowel disease; active infection; brain metastasis; synchronous or metachronous malignancy; major surgical procedure within 28 days of study enrollment; and any other cases regarded as inadequate for enrollment by the investigator.

Pretreatment evaluation. Complete clinical and radiographic staging was performed prior to enrollment in the study. Patients underwent the following evaluations: history and physical examination; CT scan of the chest, abdomen and pelvis; chest $\mathrm{X}$-ray; colonoscopy; barium enema; endoscopic ultrasound; and pelvic MRI. A complete blood count with differential analysis, serum chemistry tests, urinalysis, electrocardiogram, carcinoembryonic antigen and carbohydrate antigen 19-9
Table I. Summary of 5-fluorouracil (5-FU)/1-leucovorin/ bevacizumab dose levels used in combination with radiation therapy.

\begin{tabular}{ccccc}
\hline Level & $\begin{array}{c}\text { Bevacizumab } \\
\left(\mathrm{kg} / \mathrm{m}^{2}\right)\end{array}$ & $\begin{array}{c}5-\mathrm{FU} \text { civ } \\
\left(\mathrm{mg} / \mathrm{m}^{2}\right)\end{array}$ & $\begin{array}{c}5-\mathrm{FU} \text { bolus } \\
\left(\mathrm{mg} / \mathrm{m}^{2}\right)\end{array}$ & $\begin{array}{c}\text { L-leucovorin } \\
\left(\mathrm{mg} / \mathrm{m}^{2}\right)\end{array}$ \\
\hline-1 & 5 & 1,600 & 400 & 200 \\
1 & 5 & 2,000 & 400 & 200 \\
2 & 5 & 2,400 & 400 & 200 \\
3 & 5 & 3,000 & 400 & 200 \\
\hline
\end{tabular}

civ, continuous intravenous infusion.

Table II. Patient characteristics.

Characteristics

Age in years (range)

$65(57-75)$

Gender

Male

4

Female

2

Performance status

0

1

Tumor site

$\mathrm{Rb}$

4

Rb-P

Clinical stage (pretreatment)

T3NOM0

T3N1M0

1

T4N1M0

1

T4N2M0

2

$\mathrm{Rb}$, rectum below the peritoneal reflection; $\mathrm{P}$, anal canal.

levels were obtained. During treatment, patients were evaluated at least weekly via a history and physical examination and they underwent a complete blood count with differential analysis, electrolyte analysis, liver function tests, chemistry panel assays, coagulation panel assays and urinalysis.

\section{Treatment}

Radiation therapy. The patients underwent the 4-field (anterior-posterior, posterior-anterior and right and left laterals) approach and radiation therapy was delivered using a 10-MV linear accelerator. Patients were treated in the prone position, using a dedicated device to minimize exposure of the small bowel. A CT-based treatment planning system was mandatory for defining the planning target volume, including the primary tumor, internal iliac lymph nodes and the surrounding mesorectum. Radiotherapy was administered in fractions of $1.8 \mathrm{~Gy} /$ day, 5 days/week, for 5 weeks. The total dose of radiation delivered was $45 \mathrm{~Gy}$. 

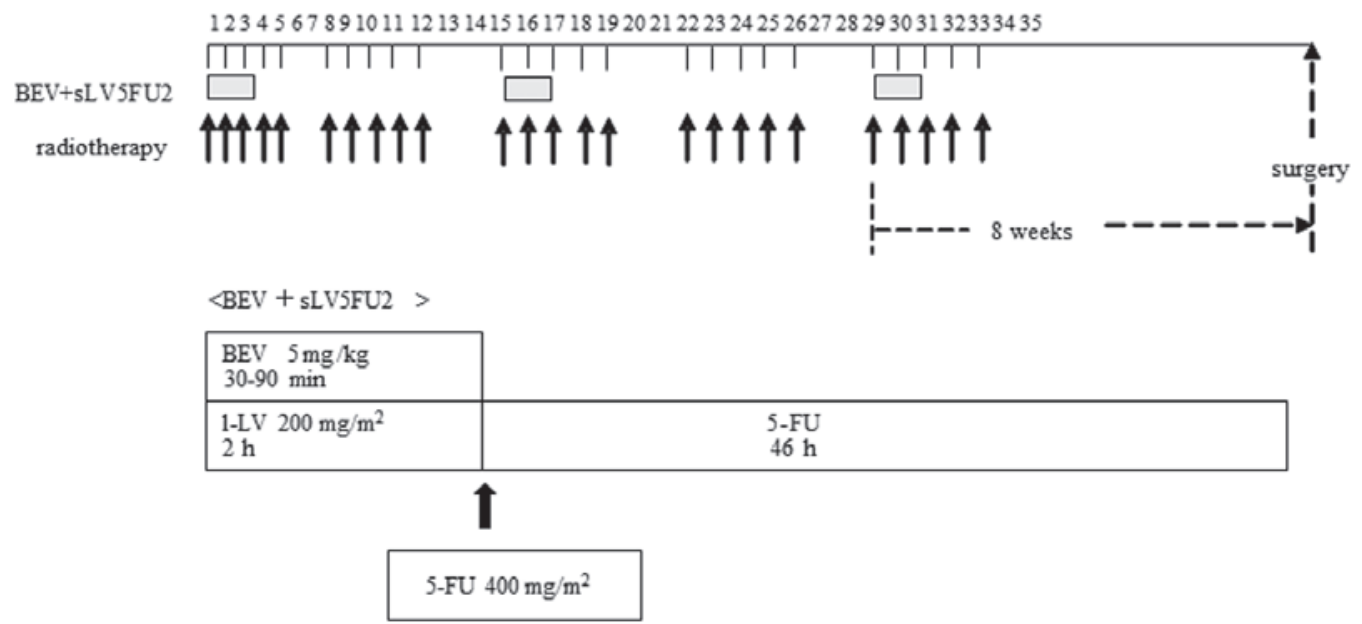

Figure 1. Concomitant chemoradiotherapy with the simplified regimen of 5-fluorouracil and 1-leucovorin (sLV5FU2) plus bevacizumab in patients with locally advanced rectal cancer. 5-FU, 5-fluorouracil; LV, 1-leucovorin; BEV, bevacizumab.

Chemotherapy. During radiation therapy, patients received three courses of the simplified $\mathrm{LV}$ and 5-FU regimen (sLV5FU2) with bevacizumab. The sLV5FU2 regimen consisted of $200 \mathrm{mg} / \mathrm{m}^{2}$ of LV, administered continuously by intravenous (i.v.) infusion over $2 \mathrm{~h}$, followed by $400 \mathrm{mg} / \mathrm{m}^{2}$ of 5-FU, administered by i.v. bolus injection, at an initial loading dose of $2,000 \mathrm{mg} / \mathrm{m}^{2}$ over $46 \mathrm{~h}$. The sLV5FU2 was administered every 2 weeks during radiotherapy and bevacizumab was infused at a fixed dose of $5 \mathrm{mg} / \mathrm{kg}$ over 30-90 min on Days 1, 15 and 29. Table I provides a summary of 5-FU dose levels delivered by continuous i.v. infusion. 5-FU administration was initiated at $2,000 \mathrm{mg} / \mathrm{m}^{2}$, with a planned dose escalation to dose levels 2 and 3. A treatment schema is shown in Fig. 1. The patients were premedicated prior to chemotherapy treatment to minimize nausea and vomiting, usually with 5-HT3 receptor antagonists.

Surgery. Six to 8 weeks following the completion of chemoradiotherapy, patients were preoperatively assessed by their respective surgeons for tumor resectability. The assessment included a history and physical examination and proctosigmoidoscopy. Following thorough exploration of the peritoneal cavity for metastases, a total mesorectal excision was performed in conjunction with a colonic J-pouch-anal anastomosis (CAA), low anterior resection (LAR) or abdominoperineal resection (APR). A diverting ileostomy was used in the patients who had undergone a sphincter-saving procedure.

Assessment. Adverse events were assessed at least weekly during radiation therapy, using the National Cancer Institute common toxicity criteria (version 3.0). Side effects were managed aggressively with standard supportive measures. Resectability was also evaluated and a complete pathological response was defined as no evidence of malignancy in the specimen.

Study design, definitions and endpoints. The primary endpoint of this study was the determination of the MTD and the RD. The secondary endpoint was evaluation of the extent and frequency of the adverse events and the resectability of locally advanced rectal cancer. The dose-limiting toxicity (DLT) was defined as Grade 4 hematological toxicity; fever with Grade 3 neutropenia; Grade 3 or higher thrombocytopenia; Grade 3 or higher non-hematological toxicity, despite adequate supportive care; any single interruption of radiation therapy of $\geq 7$ days or $>2$ interruptions per radiation course; any delay in the completion of radiation therapy of $>14$ days; and any treatment-related hospitalization or death. The MTD was defined as the dose level that produced DLT in at least two out of three patients, or two out of six patients. If DLT occurred in one of the first three patients, three additional patients were assigned to receive the same dose level. If none of the three patients initially receiving a given dose level exhibited DLT, or if one out of six patients exhibited DLT, the dose was increased to the next level. Dose escalation was not allowed in the same patient. The dose level immediately below the MTD was considered the RD for phase II studies. In this study, we also compared the adverse events of enrolled patients with those from our conventional chemoradiotherapy (sLV5FU2) treatment group.

\section{Results}

Patient characteristics. From December, 2009 through May, 2012, six patients treated at our hospital were enrolled in the study. Patient characteristics are summarized in Table II. Five patients had an ECOG performance status (PS) of 0 and one patient had a PS of 1 .

DLT and RD levels. The acute toxicities that were observed are listed in Table III. Three patients were initially enrolled at dose level 1. Two patients had perianal dermatitis (one had Grade 1 and the other Grade 2). In addition, two patients experienced diarrhea (one had Grade 2 and the other Grade 3) during week 3 of radiotherapy, prior to the third course of sLV5FU2 with bevacizumab. One patient had Grade 1 neutropenia, but there were no severe hematological toxicities. In the first three enrolled patients, chemoradiotherapy was completed according to the protocol, with the exception of one patient with refractory Grade 2 diarrhea who was able to complete radiation therapy without receiving a third course of sLV5FU2/bevacizumab. Of the first three patients enrolled in the study, one patient exhibited DLT due to 
Table III. Toxicity parameters evaluated in the study and matched control group.

\begin{tabular}{|c|c|c|c|c|c|c|c|c|}
\hline \multirow[b]{2}{*}{ Toxicity } & \multicolumn{4}{|c|}{ Dose level 1} & \multicolumn{4}{|c|}{ Control } \\
\hline & G1 & G2 & G3 & G4 & G1 & G2 & G3 & G4 \\
\hline \multicolumn{9}{|l|}{ Hematological } \\
\hline Neutropenia & 1 & 1 & 1 & 0 & 0 & 1 & 2 & 0 \\
\hline Anemia & 1 & 0 & 0 & 0 & 0 & 0 & 0 & 0 \\
\hline Thrombocytopenia & 0 & 0 & 0 & 0 & 0 & 0 & 0 & 0 \\
\hline \multicolumn{9}{|l|}{ Non-hematological } \\
\hline Nausea & 1 & 0 & 0 & 0 & 1 & 0 & 0 & 0 \\
\hline Vomiting & 0 & 1 & 0 & 0 & 0 & 0 & 0 & 0 \\
\hline Stomatitis & 0 & 1 & 0 & 0 & 1 & 0 & 0 & 0 \\
\hline Fatigue & 1 & 1 & 0 & 0 & 1 & 1 & 0 & 0 \\
\hline Diarrhea & 0 & 2 & 2 & 0 & 1 & 3 & 0 & 0 \\
\hline Skin (perianal dermatitis) & 2 & 3 & 0 & 0 & 0 & 3 & 0 & 0 \\
\hline
\end{tabular}

Table IV. Individual patient characteristics and treatment response.

\begin{tabular}{|c|c|c|c|c|c|c|}
\hline Patient & Dose level & $\begin{array}{c}\text { Pretreatment } \\
\text { stage }\end{array}$ & $\begin{array}{l}\text { Dose-limiting } \\
\text { toxicity }\end{array}$ & $\begin{array}{c}\text { Radiographic } \\
\text { response }\end{array}$ & $\begin{array}{c}\text { Pathological } \\
\text { effects }^{\mathrm{a}} \\
\text { Grade }\end{array}$ & $\begin{array}{l}\text { Surgical } \\
\text { outcome }\end{array}$ \\
\hline 1 & 1 & T3N0M0 & No & PR & 2 & R0 \\
\hline 2 & 1 & T4N1M0 & Yes & PR & $3^{\mathrm{b}}$ & $\mathrm{R} 0$ \\
\hline 3 & 1 & T4N2M0 & No & SD & $1 \mathrm{a}$ & R1 \\
\hline 4 & 1 & T4N2M0 & No & PR & $1 b$ & R1 \\
\hline 5 & 1 & T3N0M0 & Yes & SD & $1 \mathrm{a}$ & R0 \\
\hline 6 & 1 & T3N1M0 & No & PR & $3^{\mathrm{b}}$ & R0 \\
\hline
\end{tabular}

${ }^{a}$ The degree of histopathological tumor regression based on the Guidelines for Clinical and Pathological Studies on Carcinoma of the Colon and Rectum: Grade 1a,>2/3 vital residual tumor cells (VRTCs); Grade 1b, 1/3-2/3 VRTCs; Grade 2, <1/3 VRTSCs; and Grade 3 , no VRTCs. ${ }^{\mathrm{b}} \mathrm{pCR}$, pathologic complete response; PR, partial response; SD, stable disease; R1, microscopically-positive resection margin; R0, microscopically-negative resection margin.

diarrhea. Subsequently, three additional patients were enrolled at the same dose level 1. Of these additional three patients, the first patient had Grade 2 neutropenia, Grade 2 stomatitis and Grade 2 perianal dermatitis; however, chemoradiotherapy was completed according to the protocol. The second patient had Grade 2 vomiting, Grade 3 diarrhea and Grade 2 perianal dermatitis during week 4 and required hospitalization and infusion therapy, which constituted a protocol-defined DLT. Following a 1-week treatment interruption, the patient was able to complete radiotherapy, without receiving a final course of sLV5FU2/bevacizumab. The third patient developed Grade 3 neutropenia and Grade 2 diarrhea during Week 2 of radiotherapy. Although the second course of sLV5FU2/bevacizumab was postponed for 1 week, the patient was able to complete chemoradiotherapy without receiving a final course of chemotherapy. None of the six patients experienced Grade 4 toxicities. Two out of the six patients exhibited DLT, therefore, level 1 was designated as the MTD. To determine the LD, toxicities were also compared with those of six patients who had received standard preoperative chemoradiotherapy with sLV5FU2 during the same period. The sLV5FU2 regimen of the control group consisted of $200 \mathrm{mg} / \mathrm{m}^{2}$ of $\mathrm{LV}$, administered by continuous i.v. infusion over $2 \mathrm{~h}$, followed by $400 \mathrm{mg} / \mathrm{m}^{2}$ of 5-FU, administered by i.v. bolus injection at a total dose of $2,400 \mathrm{mg} / \mathrm{m}^{2}$ over $46 \mathrm{~h}$; the total dose of radiation delivered was 45 Gy. Patient characteristics of the control group were matched for the study. In the control group, two patients had Grade 3 neutropenia, but the others had no toxicities $\geq$ Grade 3 . Frequently occurring toxicities were diarrhea in four patients (one of Grade 1 and three of Grade 2) and there were three patients with Grade 3 perianal dermatitis. There were no significant differences in toxicity between the study group and the control group. Therefore, dose level 1 was determined to have acceptable toxicity as the LD and was declared the recommended phase II dose.

Efficacy measures. The patients were evaluable for tumor response. Four out of the six patients exhibited a radiographic 
partial response as their best response and two exhibited radiographically stable disease. Details of treatment response for the patients are presented in Table IV. Laparotomy was performed in the patients. One patient underwent LAR with colonic J-pouch reconstruction, four underwent CAA and one underwent APR. The postoperative complications were perineal wound infection in one patient $(16.7 \%)$, pelvic abscess in one patient $(16.7 \%)$ and pouch-vaginal fistula in one patient $(16.7 \%)$. With regard to surgical curability, two patients had a microscopically-positive resection margin (R1) and four patients had complete resection (R0). Two patients exhibited a pathological complete response (pCR rate, 33.3\%) and one exhibited a high pathological response.

\section{Discussion}

Conventional chemoradiotherapy using 5-FU has been the standard treatment for locally advanced rectal cancer, even after several active agents, such as oxaliplatin, irinotecan and molecular-targeted agents, have been developed. To improve oncological outcome, more synergistically active and safe chemotherapy is required for administration in combination with preoperative radiotherapy, for the treatment of advanced rectal cancer. Among the established agents for recurrent or metastatic colorectal cancer, VEGF-targeted chemotherapy appears appropriate for inclusion in new chemoradiotherapeutic regimens for rectal cancer, since the radiation-enhancing effects of VEGF-targeted therapy may occur through several mechanisms, such as the normalization of tumor vascularity and subsequent reoxygenation, which results in the enhancement of the radiosensitivity of cancer cells; the killing of endothelial cells in the tumor vasculature, which results in cancer cell death; and the reduction in the number of blood endothelial and progenitor cells. However, the role of molecular-targeted agents, including bevacizumab, in preoperative chemoradiotherapy for rectal cancer remains to be elucidated.

Several lines of evidence have suggested that the inhibition of VEGF may also be effective in chemoradiotherapy for locally advanced rectal cancer. Anti-VEGF antibody has been reported to enhance the effectiveness of radiotherapy by reducing tumor vascular density and interstitial fluid pressure (IFP) in xenografts (14). It has also been reported that a single infusion of bevacizumab decreased tumor perfusion, vascular volume, microvascular density, IFP and the number of viable circulating endothelial and progenitor cells and increased the fraction of vessels with pericyte coverage in rectal cancer patients (15). Clinically, Giralt et al (16) have demonstrated that VEGF overexpression is an indicator of poor clinical outcome following preoperative chemoradiotherapy, whereas Nozue et al (17) described an association between post-treatment VEGF overexpression and distant metastases. As regards pre-treatment elevated VEGF expression, Toiyama et al (11) demonstrated that analyzing VEGF expression levels in rectal cancer may be of benefit in estimating the effects of preoperative chemoradiotherapy and predicting systemic recurrence following rectal cancer surgery.

Although several trials of bevacizumab with chemoradiotherapy have shown promising results (18-20), there is no available randomized study and there is bias associated with these single-arm studies. Willett et al (15) reported on a phase I/II study, in which 32 patients were treated with bevacizumab and 5-FU (at a dose of $225 \mathrm{mg} / \mathrm{m}^{2}$ per $24 \mathrm{~h}$ ). Radiation was delivered to the pelvis $(50.4 \mathrm{~Gy}$ in 28 fractions over 5.5 weeks) and surgery was performed 7-10 weeks following completion of the preoperative therapy. In the phase I portion of the study, two out of every five patients treated with $10 \mathrm{mg} /$ $\mathrm{kg}$ of bevacizumab developed DLT (diarrhea and colitis) and the phase II dose was reduced to $5 \mathrm{mg} / \mathrm{kg}$. The majority of the adverse effects of this regimen were mild (Grade 1/2); however, certain patients experienced Grade 3 toxicities, including diarrhea, hypertension and radiation dermatitis. Among these patients, the pCR rate was $16 \%$ and $40 \%$ of patients developed postoperative complications $(18,19)$. Recently, Crane et al $(20)$ treated 25 advanced rectal cancer patients with radiotherapy at a total dose of $50.4 \mathrm{~Gy}$, capecitabine and bevacizumab (at a dose of $5 \mathrm{mg} / \mathrm{kg}$, administered every 2 weeks) followed $\sim 7$ weeks later by total mesorectal excision. No patient experienced Grade 3 hand-foot syndrome, gastrointestinal toxicity or significant hematological toxicity. The patients received surgery and the pCR rate was $32 \%$.

In our study, we used a sLV5F2 plus bevacizumab regimen as concurrent chemotherapy. The sLV5FU2 plus bevacizumab regimen has been established as the initial therapy for patients not considered appropriate for intensive therapy, in accordance with the National Comprehensive Cancer Network guidelines, since the addition of bevacizumab to 5-FU/LV has improved the survival of patients with metastatic colorectal cancer, without the severe adverse effects that result from the use of the irinotecan- or oxaliplatin-based regimens (21). The concurrent administration of sLV5FU2 and bevacizumab causes less cytotoxicity, as well as greater oncological benefit. Therefore, this regimen seems to be appropriate for administration in combination with preoperative radiotherapy for the treatment of locally advanced rectal cancer. We infused bevacizumab at a fixed dose of $5 \mathrm{mg} / \mathrm{kg}$, according to a previous report by Willett et al (18), then evaluated the MTD and RD of 5-FU administered by continuous i.v. infusion initiated at a dose of $2,000 \mathrm{mg} / \mathrm{m}^{2}$, with a planned dose escalation. Japanese patients, who received preoperative chemoradiotherapy with the combination regimen of sLV5FU2 and bevacizumab, exhibited good compliance without compromising their quality of life and were able to receive treatment according to the planned protocol. In addition, two out of the six patients (33.3\%) exhibited pCR, despite one patient experiencing DLT, thus the final course of chemotherapy was cancelled. These results suggest that bevacizumab exerted an additional beneficial effect on positive oncological outcome in patients who underwent standard preoperative chemoradiotherapy using 5-FU-based chemotherapy.

In conclusion, the combination of 5-FU, LV, bevacizumab and radiation therapy for the treatment of locally advanced rectal cancer, as evaluated by our study, resulted in acceptable toxicity and promising effectiveness. A recommended phase II dose of sLV5FU2/bevacizumab based on our findings would consist of $200 \mathrm{mg} / \mathrm{m}^{2}$ of LV, administered by continuous i.v. infusion over $2 \mathrm{~h}$, followed by $400 \mathrm{mg} / \mathrm{m}^{2}$ of 5 -FU, administered by i.v. bolus injection at a total dose of $2,000 \mathrm{mg} / \mathrm{m}^{2}$ over $46 \mathrm{~h}$ and $5 \mathrm{mg} / \mathrm{kg}$ of bevacizumab on Days 1, 15 and 29, in combination with radiation therapy. A phase II study should be performed to examine the efficacy and safety of our regimen in patients with locally advanced rectal cancer. 


\section{References}

1. Douglass HO Jr, Moertel CG, Mayer RJ, et al: Survival after postoperative combination treatment of rectal cancer. N Engl J Med 315: 1294-1295, 1986.

2. Cammà C, Giunta M, Fiorica F, et al: Preoperative radiotherapy for resectable rectal cancer: a meta-analysis. JAMA 284: 1008-1015, 2000.

3. Bosset JF, Collette L, Calais G, et al: Chemotherapy with preoperative radiotherapy in rectal cancer. N Engl J Med 355: $1114-1123,2006$.

4. Gérard JP, Conroy T, Bonnetain F, et al: Preoperative radiotherapy with or without concurrent fluorouracil and leucovorin in T3-4 rectal cancers: results of FFCD 9203. J Clin Oncol 24: 4620-4625, 2006.

5. Sato T, Ozawa H, Hatate K, et al: A phase II trial of neoadjuvant preoperative chemoradiotherapy with S-1 plus irinotecan and radiation in patients with locally advanced rectal cancer: clinical feasibility and response rate. Int J Radiat Oncol Biol Phys 79: 677-683, 2011.

6. Rödel C, Liersch T, Hermann RM, et al: Multicenter phase II trial of chemoradiation with oxaliplatin for rectal cancer. J Clin Oncol 25: 110-117, 2007.

7. Mohiuddin M, Winter K, Mitchell E, et al: Randomized phase II study of neoadjuvant combined-modality chemoradiation for distal rectal cancer: Radiation Therapy Oncology Group Trial 0012. J Clin Oncol 24: 650-655, 2006.

8. Cancer and Leukemia Group B 89901, Ryan DP, Niedzwiecki D, Hollis D et al: Phase I/II study of preoperative oxaliplatin, fluorouracil, and external-beam radiation therapy in patients with locally advanced rectal cancer: Cancer and Leukemia Group B 89901. J Clin Oncol 24: 2557-2562, 2006.

9. Wadlow RC and Ryan DP: The role of targeted agents in preoperative chemoradiation for rectal cancer. Cancer 116: 3537-3548, 2010.

10. Inoue Y, Ojima E, Watanabe $\mathrm{H}$, et al: Does preoperative chemo-radiotherapy enhance the expression of vascular endothelial growth factor in patients with rectal cancer? Oncol Rep 18: 369-375, 2007.

11. Toiyama Y, Inoue Y, Saigusa S, et al: Gene expression profiles of epidermal growth factor receptor, vascular endothelial growth factor and hypoxia-inducible factor- 1 with special reference to local responsiveness to neoadjuvant chemoradiotherapy and disease recurrence after rectal cancer surgery. Clin Oncol (R Coll Radiol) 22: 272-280, 2010.
12. Dipetrillo T, Pricolo V, Lagares-Garcia J, et al: Neoadjuvant bevacizumab, oxaliplatin, 5-fluorouracil, and radiation for rectal cancer. Int J Radiat Oncol Biol Phys 82: 124-129, 2012.

13. Kennecke H, Berry S, Wong R, et al: Pre-operative bevacizumab, capecitabine, oxaliplatin and radiation among patients with locally advanced or low rectal cancer: a phase II trial. Eur J Cancer 48: 37-45, 2012.

14. Kozin SV, Boucher Y, Hicklin DJ, et al: Vascular endothelial growth factor receptor-2-blocking antibody potentiates radiation-induced long-term control of human tumor xenografts. Cancer Res 61: 39-44, 2001.

15. Willett CG, Boucher Y, di Tomaso E, et al: Direct evidence that the VEGF-specific antibody bevacizumab has antivascular effects in human rectal cancer. Nat Med 10: 145-147, 2004.

16. Giralt J, Navalpotro B, Hermosilla E, et al: Prognostic significance of vascular endothelial growth factor and cyclooxygenase- 2 in patients with rectal cancer treated with preoperative radiotherapy. Oncology 71: 312-319, 2006.

17. Nozue M, Isaka N and Fukao K: Over-expression of vascular endothelial growth factor after preoperative radiation therapy for rectal cancer. Oncol Rep 8: 1247-1249, 2001.

18. Willett CG, Boucher Y, Duda DG, et al: Surrogate markers for antiangiogenic therapy and dose-limiting toxicities for bevacizumab with radiation and chemotherapy: continued experience of a phase I trial in rectal cancer patients. J Clin Oncol 23: 8136-8139, 2005.

19. Willett CG, Duda DG, di Tomaso E, et al: Efficacy, safety, and biomarkers of neoadjuvant bevacizumab, radiation therapy, and fluorouracil in rectal cancer: a multidisciplinary phase II study. J Clin Oncol 27: 3020-3026, 2009.

20. Crane CH, Eng C, Feig BW, et al: Phase II trial of neoadjuvant bevacizumab, capecitabine, and radiotherapy for locally advanced rectal cancer. Int J Radiat Oncol Biol Phys 76: 824-830, 2010.

21. Kabbinavar F, Hambleton J, Mass RD, et al: Combined analysis of efficacy: the addition of bevacizumab to fluorouracil/leucovorin improves survival for patients with metastatic colorectal cancer. J Clin Oncol 23: 3706-3712, 2005. 